\title{
Optimization of carboxymethylcellulase production from Bacillus amyloliquefaciens SS35
}

\author{
Shuchi Singh • Vijayanand S. Moholkar • \\ Arun Goyal
}

Received: 19 May 2013/ Accepted: 26 August 2013/Published online: 6 September 2013

(C) The Author(s) 2013. This article is published with open access at Springerlink.com

\begin{abstract}
In this paper, we have attempted optimization of production of enzyme carboxymethylcellulase or endoglucanase from the bacterium Bacillus amyloliquefaciens SS35. Optimization has been carried out in two stages using statistical experimental design, viz. medium optimization and optimization of fermentation parameters. For medium optimization, Plackett-Burman design followed by central composite design (CCD) was used, while for optimization of fermentation parameters one-variable-at-atime method followed by CCD was used. Carbon and nitrogen sources in the medium have been revealed to be the significant factors for enzyme production (carboxymethylcellulose $18.05 \mathrm{~g} / \mathrm{L}$; yeast extract $8 \mathrm{~g} / \mathrm{L}$ and peptone $2 \mathrm{~g} / \mathrm{L}$ ). The inorganic salts have been found to be insignificant components of medium. Optimum fermentation parameters for optimized medium were: initial medium $\mathrm{pH}$ 5.65, incubation temperature $=40{ }^{\circ} \mathrm{C}$, shaking speed $=120 \mathrm{rpm}$, and inoculum size $=6.96 \%, \quad \mathrm{v} / \mathrm{v}$. Interestingly, the influence of all four parameters was almost independent with no interlinks. Secondly, the
\end{abstract}

Electronic supplementary material The online version of this article (doi:10.1007/s13205-013-0169-6) contains supplementary material, which is available to authorized users.

S. Singh · V. S. Moholkar · A. Goyal

Center for Energy, Indian Institute of Technology Guwahati,

Guwahati 781 039, Assam, India

V. S. Moholkar ( $)$

Department of Chemical Engineering, Indian Institute of Technology Guwahati, Guwahati 781 039, Assam, India e-mail: vmoholkar@iitg.ernet.in

A. Goyal ( $\square)$

Department of Biotechnology, Indian Institute of Technology

Guwahati, Guwahati 781 039, Assam, India

e-mail: arungoyl@iitg.ernet.in overall effect of all parameters was also low, as indicated by linear, square and interaction regression coefficients that were at least one order of magnitude lower than the intercept in the model equation. These results essentially meant that medium components dominate overall enzyme production process in comparison to fermentation parameters.

Keywords Carboxymethylcellulase $\cdot$ Bacillus amyloliquefaciens $\cdot$ Medium optimization $\cdot$ Fermentation parameter optimization - Central composite design
Abbreviations
OVAT One-variable-at-a-time
CCD Central composite design
CMC Carboxymethylcellulose
CMCase Carboxymethylcellulase

\section{Introduction}

Use of cellulases in bioethanol production from various lignocellulosic substrates has been reported by many authors recently (Ballesteros et al. 2004; Kuhad et al. 2010; Mutreja et al. 2011; Rodhe et al. 2011). Cellulases essentially convert cellulose fraction of lignocellulosic biomass to fermentable sugars. Cellulases, which essentially are the suite of enzymes that act synergistically for complete hydrolysis of cellulose to glucose, include: endoglucanases (carboxymethylcellulase, E.C. 3.2.1.4), exoglucanases (avicelase, E.C. 3.2.1.91), and cellobiases ( $\beta$-glucosidase, E.C. 3.2.1.21) (Lynd et al. 2002). Among the group of cellulase enzymes, carboxymethylcellulase 
(CMCase) has been used predominantly in the process of ethanol production (Ballesteros et al. 2004; Zheng et al. 2009; Singh and Bishnoi 2012). Commercial cellulases are mainly produced by fungi (e.g., Aspergillus and Trichoderma sp.); however, bacteria have also been considered as robust and versatile enzyme producers because of their high growth rate, stability at extreme conditions, and presence of multi-enzyme complexes. Among these, Bacillus sp. has been revealed to be the most potent extracellular enzyme producer (Singh et al. 2001; Pason et al. 2006; Khan and Husaini 2006; Lee et al. 2008; Van Dyk et al. 2009; Kim et al. 2009). Enzymatic hydrolysis of biomass is an important step in the production of bioethanol. Enzyme being an expensive component of this process, the economical production of cellulase is an important aspect of the overall economic feasibility of bioethanol production. The extent of enzyme production by a bacterial culture is a function of fermentation parameters and components of the medium. Optimization of these parameters is a key factor influencing the economic feasibility of enzymatic hydrolysis of biomass prior to fermentation. In this study, the medium of Bacillus amyloliquefaciens SS35 culture was optimized for CMCase production using statistical design of experiments. This cellulolytic bacterium has been isolated by us from rhinoceros dung. The conventional optimization technique for the medium as well as fermentation parameters is one-variable-at-a-time (OVAT) method. However, this method has limitations in which it is not able to identify the combined or interactive effect of different components of the medium or different parameters of fermentation process. Statistical experimental designs such as central composite experimental design fitted with second-order model can be applied to overcome this limitation. In this study, we have addressed the matter of optimization of medium as well as fermentation parameters (including fermentation parameters like $\mathrm{pH}$, temperature, inoculum size, and shaking speed) for the B. amyloliquefaciens SS35 to have enhanced enzyme production. For the optimization of medium composition, considering large number of possible medium components, we have employed a two-stage methodology of initial screening of all components using Plackett-Burman design. This design identifies the components having most significant influence on response variable, i.e., CMCase production. This is followed by a central composite design (CCD) for identification of optimum values of the significant components identified earlier for enhanced production of enzymes. The regression coefficients and ANOVA of the second-order model also help in identifying the interaction between significant medium components. For optimization fermentation parameters, we have used the CCD design preceded by OVAT method.

\section{Materials and methods}

\section{Materials}

Carboxymethylcellulose (CMC) (low viscosity, 50-200 cP) was procured from Sigma-Aldrich (St. Louis, USA), and medium components (listed in next section) were procured from HiMedia Pvt. Ltd., India.

\section{Microorganism and culture conditions}

The bacterium used in this study, B. amyloliquefaciens SS35 (GenBank accession no.: JX674030), was isolated from rhinoceros dung (Singh et al. 2013), and the bacterial culture was maintained on nutrient agar slants. The basic (unoptimized) medium composition, used for CMCase production under unoptimized conditions, were $\mathrm{CMC}-\mathrm{Na}$ $(10 \mathrm{~g} / \mathrm{L})$, yeast extract $(5 \mathrm{~g} / \mathrm{L})$, peptone $(5 \mathrm{~g} / \mathrm{L}), \mathrm{K}_{2} \mathrm{HPO}_{4}$ $(1 \mathrm{~g} / \mathrm{L}), \mathrm{MgSO}_{4} \cdot 7 \mathrm{H}_{2} \mathrm{O}(0.2 \mathrm{~g} / \mathrm{L})$ and $\mathrm{NaCl}(1 \mathrm{~g} / \mathrm{L})$. The unoptimized fermentation conditions were incubation temperature $37^{\circ} \mathrm{C}$, initial medium $\mathrm{pH} 7.0$, shaking speed $180 \mathrm{rpm}$, and inoculum size $2 \%$, v/v (Optical density at $600 \mathrm{~nm}=1.0)$.

\section{Experiments for medium optimization}

The culture was grown in enzyme production medium ( $\mathrm{pH}$ 7.0) containing following six components: CMC-Na, yeast extract, peptone, $\mathrm{K}_{2} \mathrm{HPO}_{4}, \mathrm{MgSO}_{4} \cdot 7 \mathrm{H}_{2} \mathrm{O}$, and $\mathrm{NaCl}$. These medium components were selected on the basis on published literature on CMCase production from different Bacillus sp. (Apun et al. 2000; Singh et al. 2001; Lee et al. 2008; Kim et al. 2009; Rastogi et al. 2009; Deka et al. 2011). Two levels of concentrations of each of these components (again based on the published literature) were selected for Plackett-Burman analysis. The total number of experiments, with permutation-combination of different components and their levels, were 20. The details of these experiments are given in Table S1 in the supplementary material provided with this paper. In each of these 20 experiments, three trial runs have been taken so as to assess the reproducibility of results. In each experiment, 100-mL medium containing $2 \%\left(\mathrm{OD}_{600 \mathrm{~nm}}=1.0\right)$ inoculum was taken in a 250 -mL Erlenmeyer flask and incubated at $37{ }^{\circ} \mathrm{C}$ with shaking at $180 \mathrm{rpm}$ for $72 \mathrm{~h}$ in a shaking incubator (Model: Orbitek, Make: Scigenics Biotech). After every $6 \mathrm{~h}$, the culture broth was centrifuged at $12,000 \mathrm{~g}$ for $20 \mathrm{~min}$ at $4{ }^{\circ} \mathrm{C}$. The cell-free supernatant containing the crude enzyme was used for estimation of CMCase activity (the enzyme assay has been explained subsequently). It was observed that the CMCase activity showed maxima after $48 \mathrm{~h}$ of incubation, and a reduction in activity was seen thereafter. In view of this, the activity at $48 \mathrm{~h}$ was 
considered for analysis. The cell growth was monitored by taking OD at $600 \mathrm{~nm}$ using UV-visible spectrophotometer (Varian, Model Cary-100).

\section{Experiments for fermentation parameters optimization}

The optimization parameters in this category were: (1) incubation temperature $\left({ }^{\circ} \mathrm{C}\right),(2)$ initial $\mathrm{pH}$ of the medium, (3) shaking speed (rpm), and (4) inoculum size (\%, v/v). The optimized medium (defined subsequently) was used for further optimization of fermentation parameters, and the initial $\mathrm{pH}$ of the medium was adjusted to the selected range ( $\mathrm{pH} 4.5-11.0)$ in different sets of experiments using $2 \mathrm{~N} \mathrm{HCl}$ and $\mathrm{NaOH}$. In this case, we have adopted the CCD experimental design preceded by OVAT analysis to determine the levels of these parameters. The CCD experimental design and its analysis are based on these levels. The exact experimental design employed is described in Table S2 given as supplementary material.

\section{CMCase assay}

The response variable of the statistical experiments is CMCase activity $(\mathrm{U} / \mathrm{mL})$, which was quantified on the basis of measurement of total reducing sugar liberated after incubation of enzyme with CMC. The enzyme assay was carried out by incubating the enzyme (cell-free supernatant) with $\mathrm{CMC}$ for $15 \mathrm{~min}$ at $55{ }^{\circ} \mathrm{C}$. The reaction mixture $(100 \mu \mathrm{L})$ contained $20 \mu \mathrm{L}$ of enzyme and $1 \%(\mathrm{w} / \mathrm{v})$ final concentration of CMC in 50-mM sodium acetate buffer ( $\mathrm{pH}$ 5.0). The total reducing sugar was quantified using method of Nelson and Somogyi (Nelson 1944; Somogyi 1945). The absorbance was measured at $500 \mathrm{~nm}$ using a UV-visible spectrophotometer (Varian, Model Cary-100) against a blank with D-glucose as standard. One unit (U) of cellulase activity is defined as the amount of enzyme that liberates $1 \mu \mathrm{mol}$ of reducing sugar (glucose) in $1 \mathrm{~min}$ at $55^{\circ} \mathrm{C}$ and $\mathrm{pH}$ 5.0.

Experimental designs for medium optimization

\section{Plackett-Burman design}

As stated earlier, the Plackett-Burman experimental design was used for initial screening of medium components to short-list components having significant effect on CMCase production. This experimental design was devised using statistical software package MINITAB (Release 15.1, PA, USA, Trial Version). Plackett-Burman factorial design is a two-level fractional design that follows first-order polynomial model:
$Y=\beta_{\mathrm{o}}+\sum_{i=1}^{n} \beta_{i} X_{i}$

Notations: $Y$ response variable (CMCase activity), $\beta_{\mathrm{o}}$ model intercept, $\beta_{i}$ linear coefficient, and $X_{i}$ level of independent variable. Totally six medium components (or factors) have been examined at two levels each, viz. -1 and +1 , indicating low and high level, respectively (Plackett and Burman 1946). These components (or factors) and their coded as well as actual values (given in parentheses) are shown in Table S1. This experimental design comprised of a total of 20 sets of experiments (with each experiment conducted in triplicate to assess reproducibility). The average CMCase activity in each experiment was considered as the response. The regression analysis of Plackett-Burman design revealed the significant variables (or medium components) influencing CMCase activity, with significance level $\geq 95 \%$ and $p$ value $<0.05$ (Table $1 \mathrm{a}$ ). This result was further corroborated with CCD of experiments.

\section{Central composite design}

The central composite experimental design is commonly employed for processes with significant interaction effects between variables. This design essentially fits a secondorder polynomial model. We have used a 3-factor-5-level design, in which five coded levels $(-\alpha,-1,0,+1,+\alpha)$ were assigned to each factor. $\alpha$ is the extended level with value of $(2)^{3 / 4}=1.682$. A $2^{3}$ full-factorial CCD experimental design with three significant medium constituents (viz., CMC, yeast extract and peptone, as explained subsequently) at five coded levels was generated by Minitab statistical software (Release 15, Trial Version). This experimental design comprised of 20 individual experiments $\left(=2^{k}+2 k+n_{\mathrm{o}}\right)$, where ' $k$ ' is the number of independent variables and $n_{\mathrm{o}}$ is the number of replicate runs at center point of the variables. This design is described in Table S2.

\section{Statistical analysis and model fitting}

The experimental data (Table S2) were analyzed by the regression procedure to fit the following second-order polynomial equation:

$Y=\beta_{\mathrm{o}}+\sum_{i=1}^{k} \beta_{i} X_{i}+\sum_{i=1}^{k} \beta_{i i} X_{i}^{2}+\sum_{i \neq j} \sum_{i} \beta_{i j} X_{i} X_{j}$

Notation: $Y$ response (CMCase activity), $k$ number of factors or medium components, $\beta_{\mathrm{o}}$ regression constant, $\beta_{i}$ linear coefficient, $\beta_{i i}$ quadratic coefficient, and $\beta_{i j}$ interaction coefficient. The following equation was used for coding (in the range of -1 to +1 ) the actual experimental values (given in the parentheses in the table) of the factors: 
Table 1 Statistical analysis for the results from Plackett-Burman experimental design

\begin{tabular}{|c|c|c|c|c|c|}
\hline Model term & Coefficient estimate & Computed $t$ value & $p$ value & Confidence level (\%) & \\
\hline \multicolumn{6}{|c|}{ (a) Coefficient values, $t$ and $p$ value for each variable } \\
\hline Intercept & 0.33265 & 51.00 & $0^{*}$ & 100 & \\
\hline $\operatorname{CMC}\left(X_{1}\right)$ & 0.11625 & 17.82 & $0^{*}$ & 100 & \\
\hline Yeast extract $\left(X_{2}\right)$ & 0.04215 & 6.46 & $0^{*}$ & 100 & \\
\hline Peptone $\left(X_{3}\right)$ & 0.02675 & 4.10 & $0.001 *$ & 99.9 & \\
\hline $\mathrm{K}_{2} \mathrm{HPO}_{4}\left(X_{4}\right)$ & 0.00755 & 1.16 & 0.268 & 73.2 & \\
\hline $\mathrm{MgSO}_{4} \cdot 7 \mathrm{H}_{2} \mathrm{O}\left(X_{5}\right)$ & -0.01105 & -1.69 & 0.114 & 88.6 & \\
\hline $\mathrm{NaCl}\left(X_{6}\right)$ & 0.00755 & 1.16 & 0.268 & 73.2 & \\
\hline Variable & SS & DF & MS & $F$ value & $\begin{array}{l}p \text { value } \\
\text { prob }>F\end{array}$ \\
\hline \multicolumn{6}{|c|}{ (b) ANOVA for the model } \\
\hline Constant & 0.325 & 6 & 0.0541 & 63.62 & 0 \\
\hline $\operatorname{CMC}\left(X_{1}\right)$ & 0.27 & 1 & 0.27 & 317.59 & 0 \\
\hline Yeast extract $\left(X_{2}\right)$ & 0.0355 & 1 & 0.0355 & 41.75 & 0 \\
\hline Peptone $\left(X_{3}\right)$ & 0.0143 & 1 & 0.0143 & 16.82 & 0.001 \\
\hline $\mathrm{K}_{2} \mathrm{HPO}_{4}\left(X_{4}\right)$ & 0.00114 & 1 & 0.00114 & 1.34 & 0.268 \\
\hline $\mathrm{MgSO}_{4} \cdot 7 \mathrm{H}_{2} \mathrm{O}\left(X_{5}\right)$ & 0.00244 & 1 & 0.00244 & 2.87 & 0.114 \\
\hline $\mathrm{NaCl}\left(X_{6}\right)$ & 0.00114 & 1 & 0.00114 & 1.16 & 0.268 \\
\hline Residual & 0.0111 & 13 & 0.000851 & 1.34 & \\
\hline Total & 0.336 & 19 & & & \\
\hline
\end{tabular}

$D F$ degrees of freedom, $S S$ sum of squares, $M S$ mean square

* Significant $p$ values, $p \leq 0.05 ; R^{2}=0.9671$; predicted $R^{2}=0.9220$; adjusted $R^{2}=0.9519$

$x_{i}=X_{i}-X_{\mathrm{o}} / \Delta X_{i}, \quad i=1,2,3, \ldots, k$

where, $x_{i}$ is the dimensionless value of an independent variable, $X_{i}$ is the real value of an independent variable, $X_{\mathrm{o}}$ is the value of $X_{i}$ at the center point, and $\Delta X_{i}$ is the step change. The analysis of variance (ANOVA) was also done to determine the significance of each factor in fitted model Eq. (2), and also to determine the goodness of fit. For this purpose, the software Design Expert 7.0 (trial version) has been used. Graphical representation of the fitted polynomial Eq. (2) has been given in the form of contour plots. ANOVA of the linear, quadratic and interaction regression coefficients (i.e., $F$ value and $p$ value) has been given in Table 2. The $F$ and $p$ values essentially exhibit the individual and interactive effects of the independent variables.

Experimental design for optimization of fermentation parameters

\section{Central composite design}

A $2^{4}$ full-factorial CCD experimental design with four parameters (viz., incubation temperature, initial medium $\mathrm{pH}$, rpm or speed of orbital shaking and inoculum size) at five coded levels was generated by Minitab statistical software (Release 15, Trial Version) (Table S3 provided in supplementary material). In this 4-factor-5-level design, five coded levels $(-\alpha,-1,0,+1,+\alpha)$ were assigned to each factor, where $\alpha=(2)^{4 / 4}=2$. This experimental design comprised of 31 individual experiments $\left(=2^{k}+2 k+n_{\mathrm{o}}\right)$, where $k$ is the number of independent variables (4) and $n_{\mathrm{o}}$ being the number of replicate runs (7) at center point of the variables.

Experimental validation of optimization

Experiments have been conducted in two steps with optimized medium composition and optimized fermentation parameters predicted by statistical analysis to assess the accuracy of the models. Initially, validation of optimization of medium composition was carried out using results of CCD experimental design. Next, validation of optimum fermentation parameters (at optimum medium composition) has been carried out. To assess the reproducibility, all validation experiments have been performed in triplicate.

\section{Results and discussion}

As noted earlier, we have carried out optimization of CMCase production by the bacterium B. amyloliquefaciens SS35 in two stages, viz. optimization of the medium, and 
Table 2 Results of statistical (CCD) analysis for medium optimization

\begin{tabular}{|c|c|c|c|c|c|c|}
\hline \multicolumn{2}{|l|}{ Model term } & Coefficient estimate & Computed $t$ value & $\begin{array}{l}p \text { value } \\
\text { prob }>F\end{array}$ & \multicolumn{2}{|c|}{ Confidence level (\%) } \\
\hline \multicolumn{7}{|c|}{ (a) Model coefficient estimated by regressions } \\
\hline \multicolumn{2}{|c|}{ Intercept } & 0.443596 & 56.21 & $0 *$ & \multicolumn{2}{|l|}{100} \\
\hline \multicolumn{2}{|l|}{$\operatorname{CMC}\left(X_{1}\right)$} & 0.087215 & 16.657 & $0 *$ & \multicolumn{2}{|l|}{100} \\
\hline \multicolumn{2}{|l|}{ Yeast extract $\left(X_{2}\right)$} & -0.028983 & -5.535 & $0^{*}$ & \multicolumn{2}{|l|}{100} \\
\hline \multicolumn{2}{|l|}{ Peptone $\left(X_{3}\right)$} & -0.008664 & -1.655 & 0.129 & \multicolumn{2}{|l|}{87.1} \\
\hline \multicolumn{2}{|c|}{$\mathrm{CMC} \times \mathrm{CMC}\left(X_{1}^{2}\right)$} & -0.045706 & -8.967 & $0 *$ & \multicolumn{2}{|l|}{100} \\
\hline \multicolumn{2}{|c|}{ Yeast extract $\times$ yeast extract $\left(X_{2}^{2}\right)$} & 0.008741 & 1.715 & 0.117 & \multicolumn{2}{|l|}{88.3} \\
\hline \multicolumn{2}{|c|}{ Peptone $\times$ peptone $\left(X_{3}^{2}\right)$} & -0.011942 & -2.343 & $0.041^{*}$ & \multicolumn{2}{|l|}{95.9} \\
\hline \multicolumn{2}{|c|}{$\mathrm{CMC} \times$ yeast extract $\left(X_{1} \times X_{2}\right)$} & 0.0175 & 2.558 & $0.028 *$ & 97.2 & \\
\hline $\mathrm{CMC} \times$ peptone & & 0.02175 & 3.179 & $0.01 *$ & 99 & \\
\hline Yeast extract $\times 1$ & & 0.02975 & 4.349 & $0.001 *$ & 99.9 & \\
\hline Source & $\mathrm{DF}$ & SS & MS & & $F$ value & $\begin{array}{l}p \text { value } \\
\text { prob }>F\end{array}$ \\
\hline (b) ANOVA for q & & & & & & \\
\hline Regression & 9 & 0.163 & 0.0182 & & 48.51 & 0 \\
\hline Linear & 3 & 0.116 & 0.0388 & & 103.61 & 0 \\
\hline Square & 3 & 0.0338 & 0.0113 & & 30.05 & 0 \\
\hline Interaction & 3 & 0.0133 & 0.00444 & & 11.85 & 0.001 \\
\hline Residual (error) & 10 & 0.00374 & 0.000374 & & & \\
\hline Lack of fit & 5 & 0.00281 & 0.000561 & & 2.99 & 0.127 \\
\hline Pure error & 5 & 0.000938 & 0.000188 & & & \\
\hline Total & 19 & 0.167 & & & & \\
\hline
\end{tabular}

$D F$ degrees of freedom, $S S$ sum of squares, $M S$ mean square

* Significant $p$ values, $p \leq 0.05 ; R^{2}=0.9776$; predicted $R^{2}=0.8642$; adjusted $R^{2}=0.9575$

secondly, optimization of the fermentation parameters. In accordance with this, we first present the results of medium optimization followed by results of optimization of fermentation parameters. Finally, we try to link the results of these two studies.

Optimization of fermentation medium

\section{Plackett-Burman design for screening of significant medium components}

The results of initial screening of medium components using Plackett-Burman design are given in Table S1. The experimental and predicted values of CMCase activity match quite well. The CMCase activity in the cell-free supernatant of B. amyloliquefaciens SS35 varied from 0.132 to $0.528 \mathrm{U} / \mathrm{mL}$. As stated in previous section, this is the maximum activity attained after $48 \mathrm{~h}$ of incubation. The statistical analysis of the Plackett-Burman design is given in Table 1a, b. The values of the first-order model coefficients for all six variables along with $t$ value, $p$ value, and confidence levels are depicted in Table 1a, while the ANOVA of the model is given in Table 1b. The model variable is termed to be significant if the $t$ value is greater than $p$ value. As seen from Table $1 \mathrm{a}$, this condition is satisfied by three variables, viz CMC, yeast extract, and peptone. The significance of these variables is also corroborated by results of Table $1 \mathrm{~b}$, which shows high $F$ value and zero $p$ value for these variables. The $t$ value limit for this analysis is 2.16 (as shown in Pareto chart Figure S1, given in supplementary material). The $t$ value for CMC, yeast extract, and peptone are higher than this limit, which points to their significance. Moreover, $\mathrm{MgSO}_{4} \cdot 7 \mathrm{H}_{2} \mathrm{O}$ has shown a negative effect on the CMCase activity as indicated by the negative Plackett-Burman model coefficient. Another measure of significance level of variables is the confidence level, which is $\sim 100 \%$ for CMC, yeast extract, and peptone. The overall regression coefficient for the Plackett-Burman design is $R^{2}=0.9671$, with adjusted $R^{2}=0.9519$ shows the model fits very well to the data. Neglecting the insignificant variables, the model equation for CMCase activity is written as: 
$Y_{\text {activity }}=0.33265+0.11625 X_{1}+0.04215 X_{2}+0.02675 X_{3}$

Among the significant variables $\mathrm{CMC}$ acts as carbon source, while yeast extract and peptone are the nitrogen sources. Earlier studies by Li et al. (2008) and Deka et al. (2011) report significant role played by these components on cellulase production by Bacillus sp. The inorganic salts have been found to have insignificant effect on CMCase production. This is rather an anomaly as potassium phosphate is known for the production of microbial polysaccharides and also as an ingredient in buffer solutions that enhance cell growth (Lee et al. 2008; Jin et al. 2011; Gao et al. 2012); sodium chloride as well as magnesium sulphate in the medium play important role in initial cell growth. We explain this anomaly in terms of differences in the phase of cell life cycle in which cell growth and enzyme production occurs. The maximum CMCase production was observed at late log phase or early stationary phase of life cycle (Singh et al. 2013).

\section{Optimization by central composite experimental design}

The CCD was based on the results of Plackett-Burman design, which revealed that three factors, viz. CMC, yeast extract, and peptone had significant influence on CMCase production. The full factorial CCD matrix of these variables is given in Table S2 supplementary material provided. Table $\mathrm{S} 2$ also gives the CMCase activity obtained in each experiment and the standard deviation. The results of second-order response model fitted to the coded data are given in Table 2a. The second-order regression equation fitted to this data is as follows:

$$
\begin{aligned}
Y_{\text {activity }}= & 0.443596+0.087215 X_{1}-0.028983 X_{2} \\
& -0.008664 X_{3}-0.045706 X_{1}^{2} \\
& +0.008741 X_{2}^{2}-.011942 X_{3}^{2} \\
& +0.0175 X_{1} X_{2}+0.02175 X_{1} X_{3} \\
& +0.02975 X_{2} X_{3}
\end{aligned}
$$

Notations are same as that for Plackett-Burman design. The overall model had regression coefficient of 0.9776 with adjusted coefficient of $R^{2}=0.9575$. The predicted $R^{2}$ of 0.8642 is also in reasonable agreement with adjusted $R^{2}$ of 0.9575 . This essentially indicates that the model fits very well to the experimental data. This is also corroborated by the predicted CMCase activity values (listed in Table S2 of supplementary material) that match well with the experimental values. The ANOVA of the fitted model is described in Table 2b. The lack of fit $F$ value of 2.99 implies that lack of fit is not significantly relative to pure error. The $p$ value of 0.127 for lack of fit implies that there is $12.7 \%$ chance that a 'lack of fit $F$ value' this large could occur due to noise.
Another yardstick for fitness of model is Adeq Precision value that measures the signal-to-noise ratio. A ratio $>4$ is desirable. The ratio obtained for the present analysis is 25.538, which represents adequacy of the signal. The $p$ value of the linear, square and interaction coefficients are all zero pointing their significance. An interesting trend is in the $F$ values of the coefficients. The $F$ value for linear coefficients is far higher $(>10 \times)$ than the value for interaction coefficients. This essentially means that effects of $\mathrm{CMC}$, yeast extract and peptone on the CMCase production are rather independent, with no interlinks between them.

The optimum value of concentration of medium components as revealed from the statistical analysis and quadratic model for maximum CMCase production are $\mathrm{CMC}=19.05 \mathrm{~g} / \mathrm{L}$, yeast extract $=8 \mathrm{~g} / \mathrm{L}$, and peptone $=2 \mathrm{~g} / \mathrm{L}$. These have been depicted in the desirability function plot shown in Figure S2 of supplementary material. The maximum CMCase activity for these parameters the model predicts $0.585 \mathrm{U} / \mathrm{mL}$. The experiments were conducted to validate these predictions and the CMCase activity for the optimum medium composition was found to be $0.553 \pm 0.021 \mathrm{U} / \mathrm{mL}$, which is in close agreement with predicted result of CCD. The CMCase activity under unoptimized conditions (as mentioned in section Microorganism and culture conditions) was $0.161 \pm 0.014 \mathrm{U} / \mathrm{mL}$; thus, the resulted enhancement in enzyme activity after medium optimization was about $3 \times$.

\section{Interaction effects of medium components}

The interactive effects between medium components have been assessed using contour plots shown in Fig. 1. Following trends in interactive effect can be identified: (1) for smaller concentration of both yeast extract and CMC, the enzyme activity increases with concentration. However as the $\mathrm{CMC}$ concentration crosses $16 \mathrm{~g} / \mathrm{L}$, the highest activity is seen for low concentration of yeast extract $\sim 8 \mathrm{~g} / \mathrm{L}$. (2) The highest activity is seen for the yeast extract concentration of $8 \mathrm{~g} / \mathrm{L}$ and peptone concentration range of 3-6 g/L. With concentration of peptone and yeast extract increasing beyond this range, the activity reduces. (3) The CMCase activity increases monotonically with both peptone and CMC concentration.

We explain these results on the basis of amino acid composition of the enzyme CMCase (endoglucanase) given in supplementary material. On the basis of the methodology given by Unrean and Nguyen (2013), we have also listed (Table S4 in supplementary material) the metabolic and energy requirement to produce one mole of CMCase by $B$. amyloliquefaciens. Based on the amino acid composition it could be seen that one mole of CMCase production requires large amount of nitrogen, i.e., $677 \mathrm{~mol}$ of ammonia. Consequently, both peptone and yeast extract 
being the nitrogen sources play a vital role in CMCase production. CMC being the main substrate of the metabolic pathway enhances bacterial cell growth, and thus, a significant component of the process.

\section{Optimization of fermentation parameters}

As noted earlier, the optimization of fermentation parameters was carried out in two steps: (1) OVAT method, and (2) central composite experimental design. As reported in literature (for example, Immanuel et al. 2006), the cellulase production is a function of several parameters such as temperature, initial $\mathrm{pH}$ of the medium, and inoculum size. The OVAT experiments helped in arrangement of individual effect of the fermentation parameters, as well as in deciding the levels of these parameters for a CCD design. The results of OVAT experiment are depicted in Fig. 2. It could be seen that each of the parameters shows an optimum value at which maxima of CMCase activity is observed. The values of these parameters are (1) temperature $=40{ }^{\circ} \mathrm{C},(2)$ initial $\mathrm{pH}$ of medium $=6.0$, (3) shaking speed $=150 \mathrm{rpm}$, and (4) inoculum volume $=6 \% \mathrm{v} / \mathrm{v}$.

\section{Optimization of fermentation parameters by $C C D$}

The CCD experimental matrix with the actual and coded values of the above parameters is given in Table S3 provided in supplementary material, along with the values of
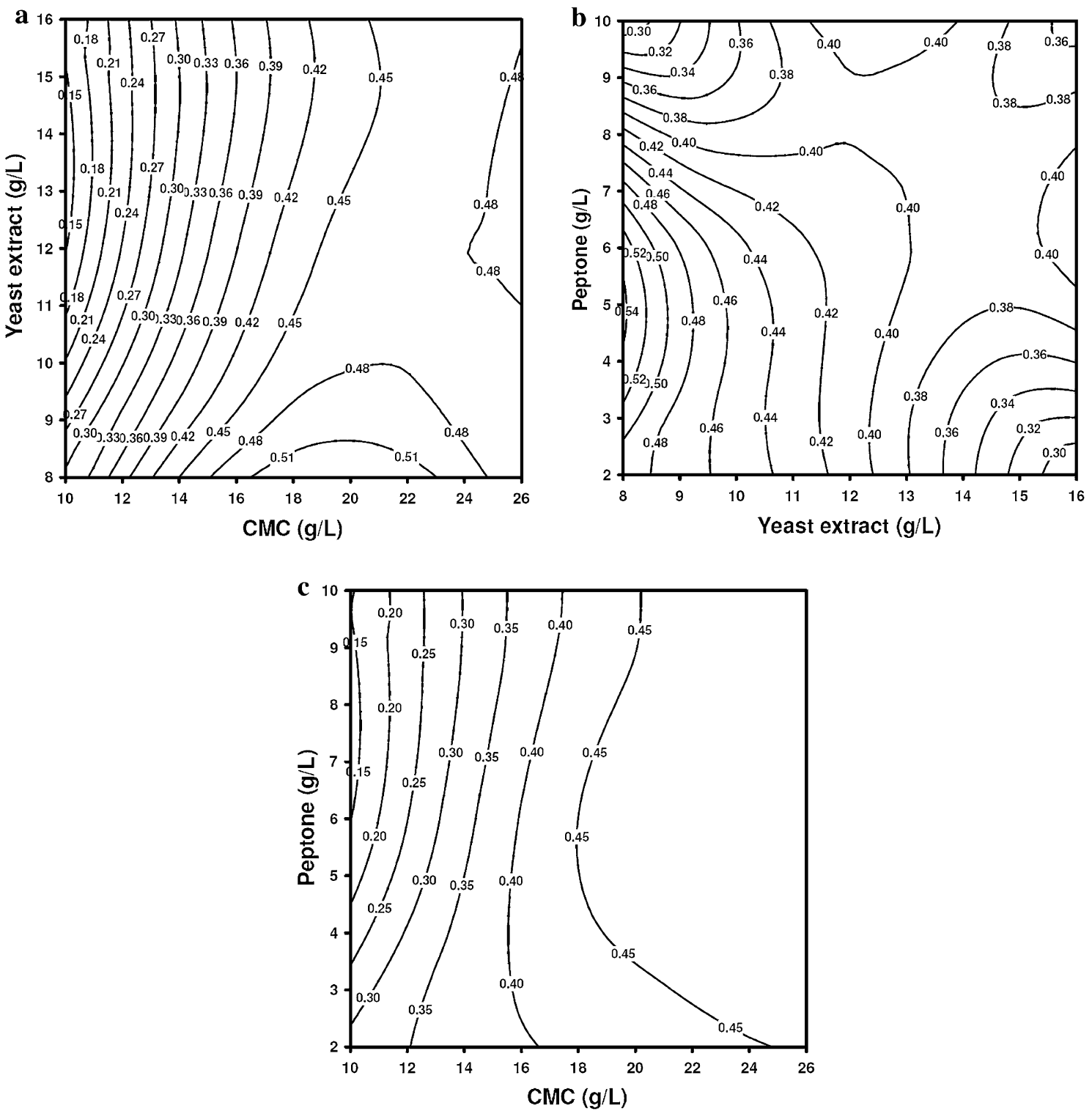

Fig. 1 Contour plots for CMCase production showing the interactive effects of medium components: a concentrations of yeast extract and CMC, b concentrations of yeast extract and peptone, c concentrations of CMC and peptone 
response variable for each experiment with different combination of parameters. The results of second-order response model fitted to the coded data and the ANOVA of the model is given in Table $3 a, b$. The model equation for response variable is as follows:

$$
\begin{aligned}
Y_{\text {activity }}= & 0.637714+0.008208 X_{1}-0.021208 X_{2} \\
& -0.006125 X_{3}-0.004958 X_{4}-0.028564 X_{1}^{2} \\
& -0.007314 X_{2}^{2}+0.008811 X_{3}^{2}-0.002689 X_{4}^{2} \\
& +0.003563 X_{1} X_{2}-0.007438 X_{1} X_{3} \\
& +0.005938 X_{1} X_{4}+0.001 X_{2} X_{3} \\
& +0.000313 X_{2} X_{4}+0.000063 X_{3} X_{4}
\end{aligned}
$$

It could be seen that all coefficients (linear/quadratic/ interaction) are at least one to three orders of magnitude smaller than the intercept value. The analysis of coefficients presented in Table 3a shows that most of linear and quadratic coefficients are significant (as indicated by $p<0.05$ ), while most of interaction coefficients are insignificant. The overall model fits very well to the data as seen from $R^{2}$ value of 0.9704 and fairly matching values of
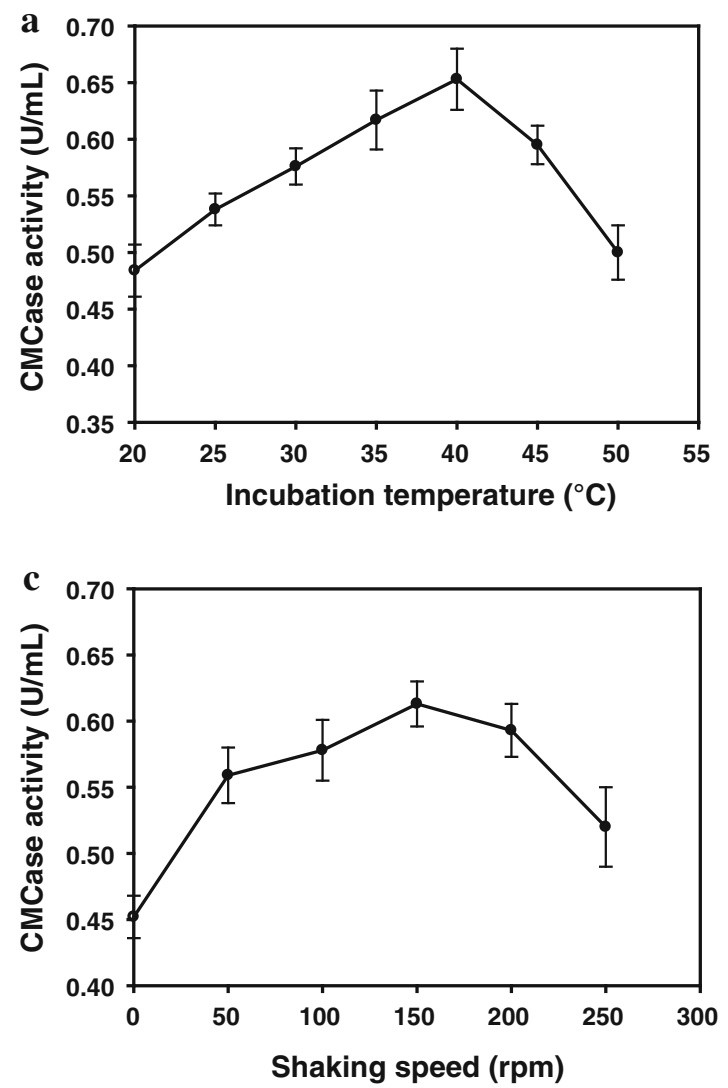

Fig. 2 Evaluation of individual effect of fermentation parameters on CMCase production (one-variable-at-a-time method): a effect on incubation temperature at medium $\mathrm{pH} 7.0$, shaking speed $=180 \mathrm{rpm}$, and inoculum size $=2 \% \mathrm{v} / \mathrm{v} ; \mathbf{b}$ effect of initial medium $\mathrm{pH}$ at temperature $=37^{\circ} \mathrm{C}$, shaking speed $=180 \mathrm{rpm}$, and inoculum
$R^{2}=0.8494$. ANOVA of the model presented in Table $3 \mathrm{~b}$ shows all regression coefficients as significant. But relative magnitude of $F$ values of these coefficients indicates that individual influence of parameters is much higher than interactive effect.

\section{Interaction effects of fermentation parameters}

The interaction between the fermentation parameters has been explained with the help of contour plots of the regression model, shown in Fig. 3a-f. The highest CMCase activity is seen for temperature range of $35-45^{\circ} \mathrm{C}$ with corresponding $\mathrm{pH}$ range of 5.0-7.0. At a given temperature, even twofold variation of shaking speed does not give a significant rise in the activity. Similarly, in the optimum $\mathrm{pH}$ range of 6.0-7.0, shaking speed does not influence CMCase activity. Same observation holds true for variation of CMCase activity with inoculum size and $\mathrm{pH}$. For the combination of $\mathrm{pH}$ and temperature, $\mathrm{CMCase}$ activity is in the temperature range of $40-45{ }^{\circ} \mathrm{C}$ and medium $\mathrm{pH}$ of 6.0-7.0. The activity rapidly reduces as either of this
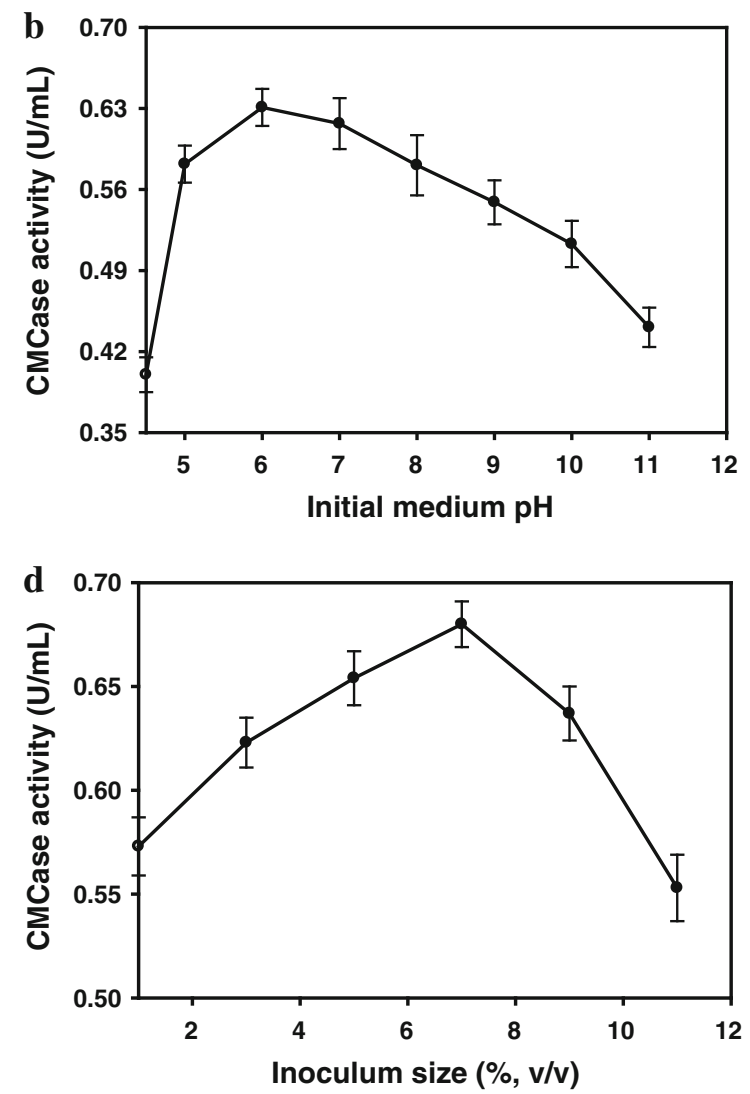

size $=2 \% \mathrm{v} / \mathrm{v} ;$ c effect of shaking speed at medium $\mathrm{pH} 7.0$, temperature $=37{ }^{\circ} \mathrm{C}$, inoculum size $=2 \% \mathrm{v} / \mathrm{v} ; \mathbf{d}$ effect of inoculum size at temperature $=37{ }^{\circ} \mathrm{C}$, shaking speed $=180 \mathrm{rpm}$, and medium pH 7.0 
Table 3 Results of statistical (CCD) analysis for optimization of fermentation parameters

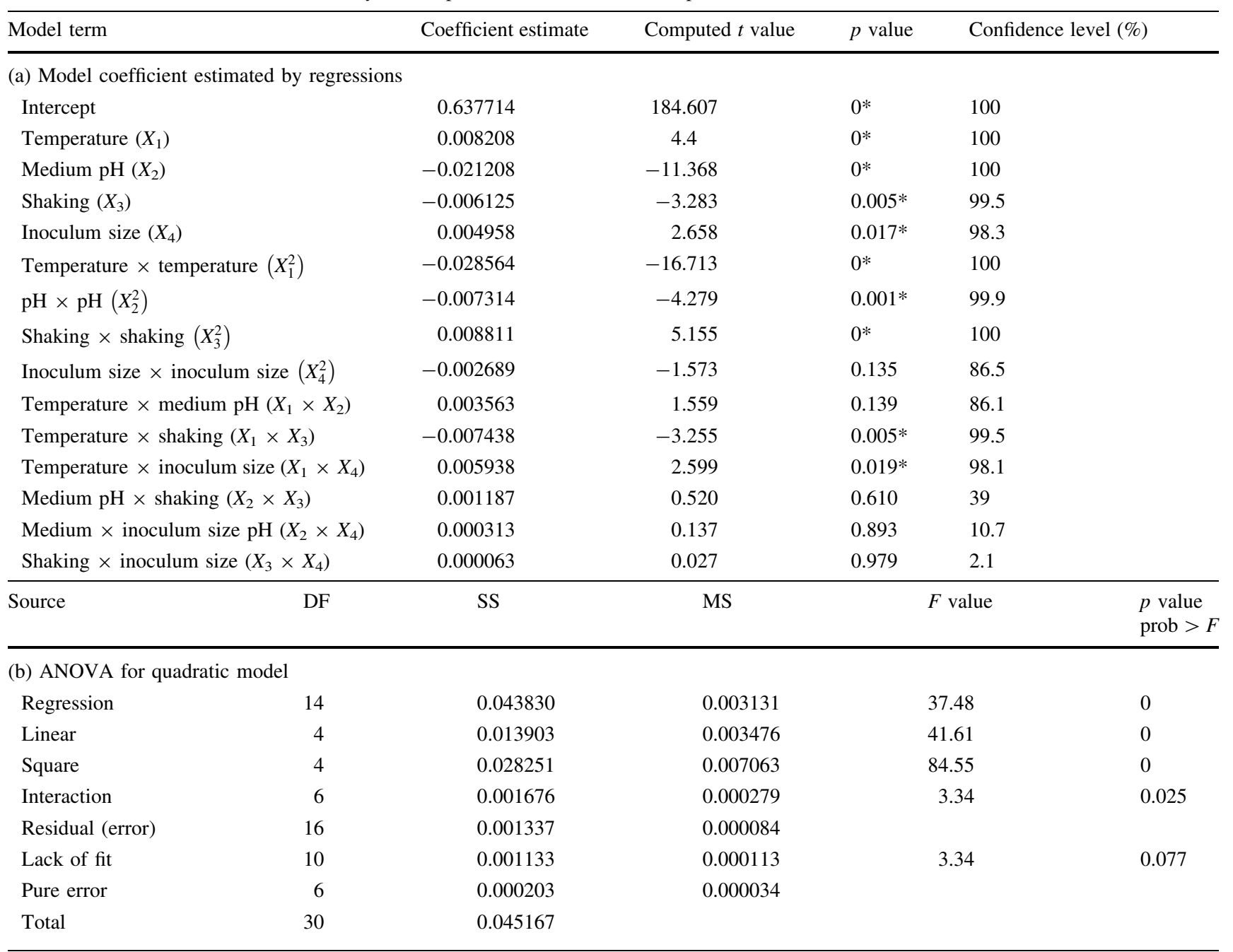

$D F$ degrees of freedom, $S S$ sum of squares, $M S$ mean square

* Significant $p$ values, $p \leq 0.05 ; R^{2}=0.9701$; predicted $R^{2}=0.9422$; adjusted $R^{2}=0.8473$

variable shift away from the optimum range. Nonetheless, an overall observation seen in all contour plots is that with twofold, threefold or even fourfold variation of each fermentation parameter; the activity shows a marginal variation of $\pm 20-40 \%$. The optimum values of the four variables that could be deduced from the desirability function plot shown in Figure S3 (in supplementary material) are temperature $=40{ }^{\circ} \mathrm{C}, \mathrm{pH} 5.65$, shaking speed $=120 \mathrm{rpm}$, and inoculum size $=6.9 \% \mathrm{v} / \mathrm{v}$. The predicted enzyme activity at these conditions was 0.710 $\mathrm{U} / \mathrm{mL}$. Validation of these results was done using shake flask experiments at the optimum values of different parameters. The experimental enzyme activity was observed to be $0.693 \pm 0.043 \mathrm{U} / \mathrm{mL}$, which is an excellent agreement with the predicted value. Quite interestingly these values match fairly well with the values obtained from OVAT analysis. The observed enhancement of CMCase activity
(0.693 U/mL) after complete optimization (i.e., medium composition and fermentation parameters) was about fourfold in comparison to activity $(0.161 \mathrm{U} / \mathrm{mL})$ under unoptimized conditions. However, the increment in activity after optimization of medium composition alone was threefold, and further optimization of fermentation parameters yielded only onefold increment. Thus, it can be concluded that the concentration of major medium components is the principal governing factor for CMCase production. The outcome of the study by Deka et al. (2013) also supports this conclusion, where total optimization of medium composition and fermentation parameters resulted to eightfold enhancement in CMCase activity, out of which the contribution of medium optimization alone to enhancement was sixfold. Optimization of CMCase production by Bacillus sp. has been studied by earlier authors using strains isolated from different sources. A 

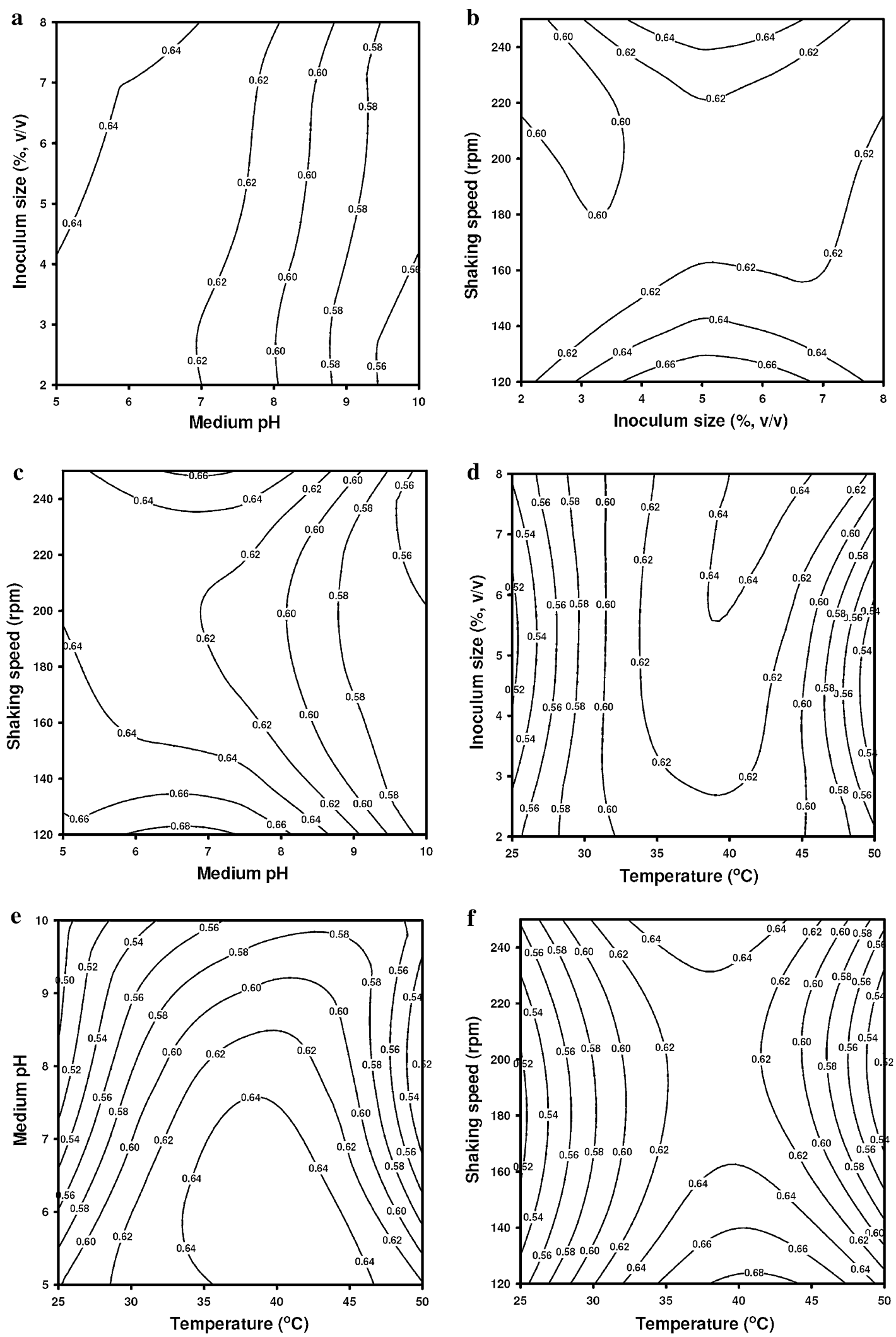

Fig. 3 Contour plot for CMCase production showing the interactive effects of fermentation parameters: a medium $\mathrm{pH}$ and inoculum size; b shaking speed and inoculum size; c shaking speed and medium $\mathrm{pH}$;

d temperature and inoculum size; e temperature and medium $\mathrm{pH}$; f temperature and shaking speed 


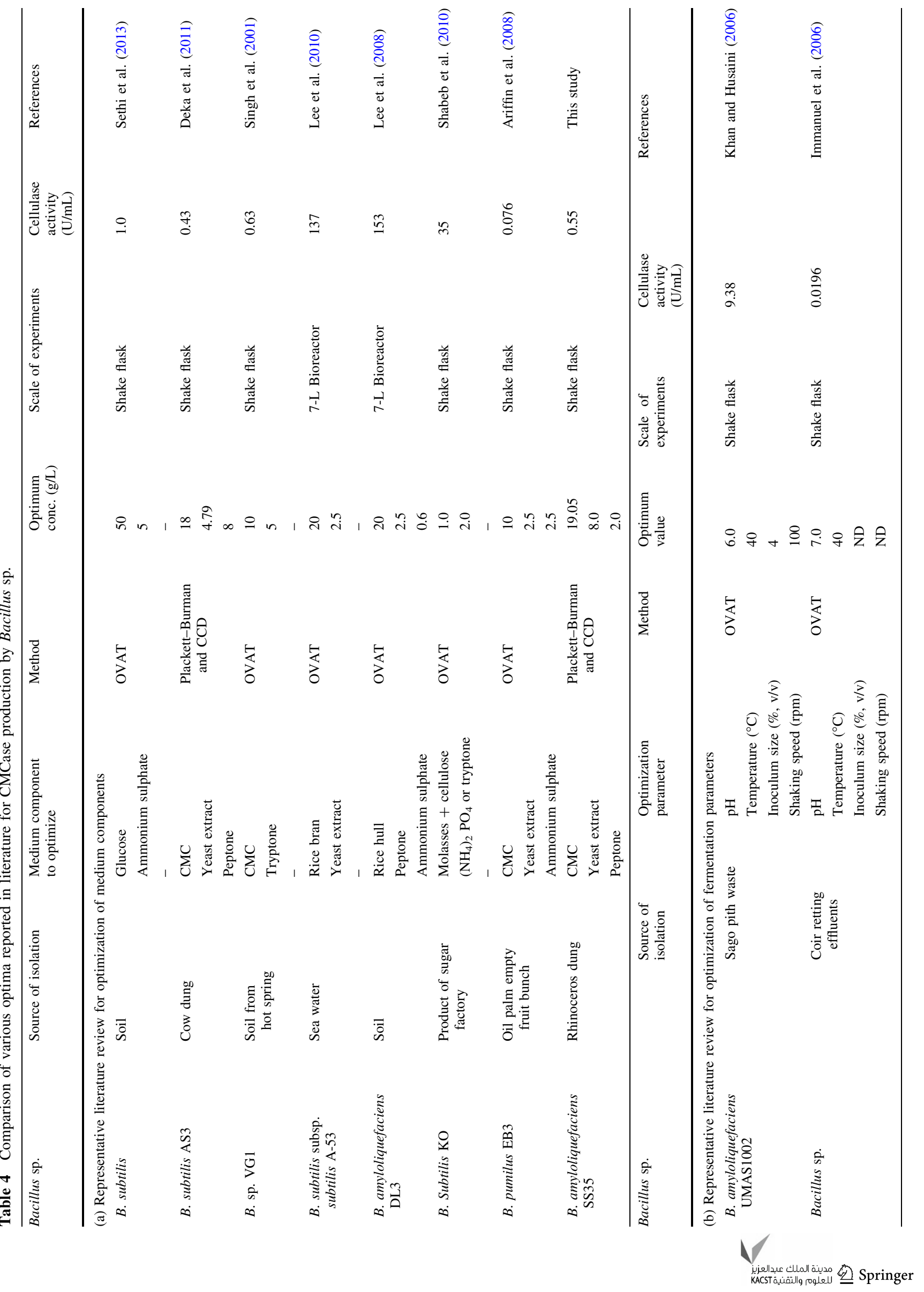




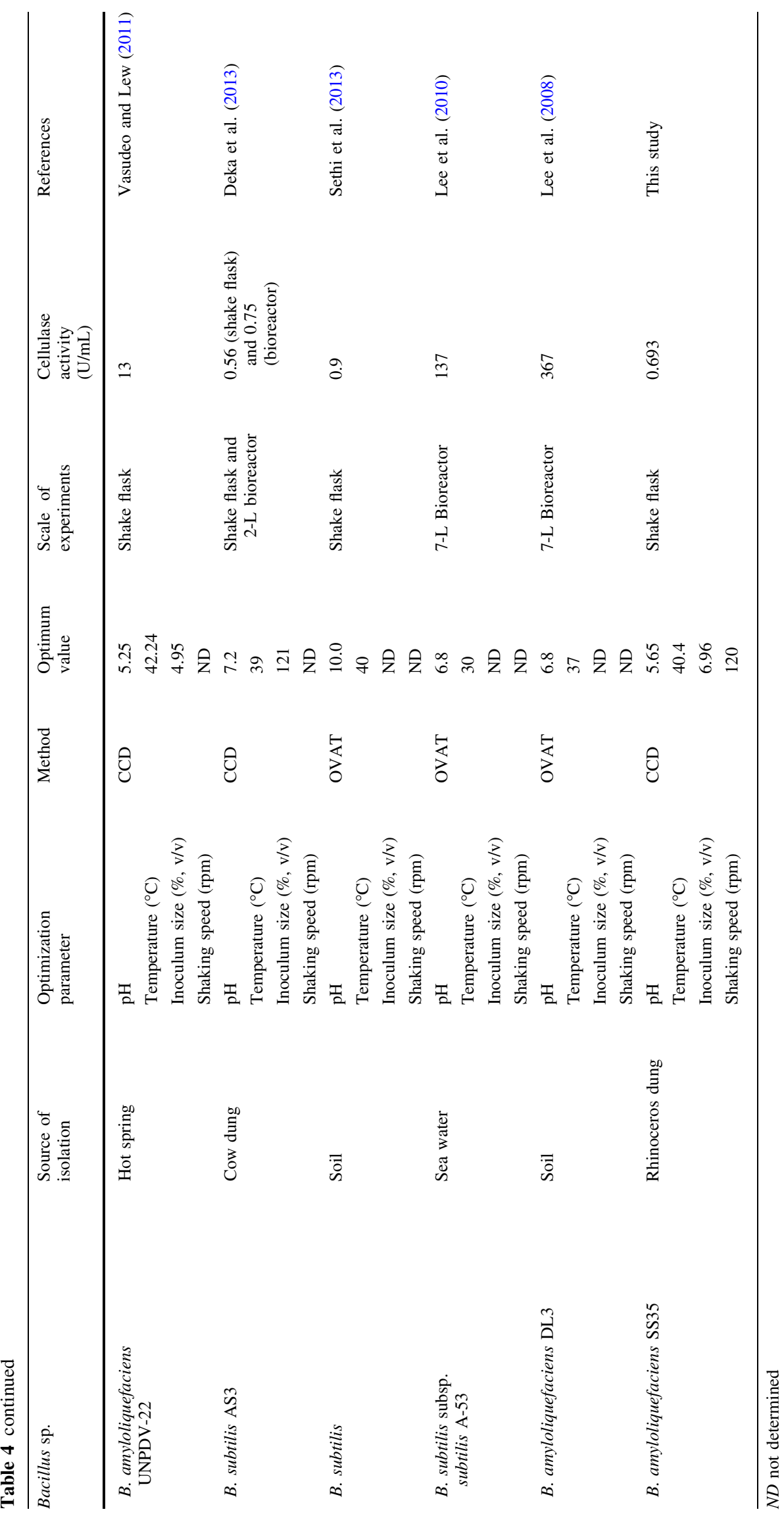


comparative account of this is given in Table $4 a, b$ for optimization of medium as well as optimization of fermentation parameters. The activity reported by Lee et al. $(2008,2010)$ for their Bacillus sp. is significantly higher (i.e., 137 and $153 \mathrm{U} / \mathrm{mL}$ ) than that reported in this study as well as several other studies cited in Table 4a. However, it should noted that the Lee et al. $(2008,2010)$ have determined the activity on bioreactor scale (as compared to shake flask scale in present study), which a far better and precisely controlled system in terms of operating parameters. Moreover, the sole carbon source for all species reported in Table 4a is different from each other, which has influence on the actual activity of the enzyme. For example, Sethi et al. (2013) have used glucose as the sole carbon source, while Shabeb et al. (2010) have used dual carbon source in the form of molasses and cellulose. It should be pointed out that bacteria producing cellulase with activity in a similar range as for our species could hydrolyze several lignocellulosic biomass significantly, depending the substrate specificity of enzyme. Examples are: (1) Wild grass (Achnatherum hymenoides) hydrolysis by Bacillus subtilis AS3 with CMCase activity $0.57 \mathrm{U} / \mathrm{mL}$ (Deka et al. 2013); (2) Sago pith waste hydrolysis by B. amyloliquefaciens UMAS 1002 with CMCase activity $0.63 \mathrm{U} / \mathrm{mL}$ (Apun et al. 2000); (3) enzymatic hydrolysis of cellulosic biomass like sugarcane bagasse for bioethanol production using cellulase with lower activity (i.e., 0.112 and 0.902 $\mathrm{U} / \mathrm{mL}$ ) produced by $T$. longibrachiatum PTCC 5140 and Aspergillus niger, respectively (Shaibani et al. 2011). We would also like to point out that Kabel et al. (2006) have proven with rigorous study of activity of numerous commercial enzyme preparations (from suppliers such as Novozymes, Dyadic, Genencor, Rhodia-Danisco, Lyven) comprising of cellulase, cellobiase, and xylanases that the activities predicted by standard assays do not really reflect the actual activity of the enzyme on all substrates. The actual activity is more substrate dependent-in that enzymes showing lower activity with standard assays can do efficient hydrolysis with a particular substrate. For convenience of the reader, we briefly reproduce here the overall outcome of the study of Kabel et al. (2006). Activity analyzed in the standard xylanase test did not present a high correlation with the degradation of xylans in the xylan-rich KOHss fractions of wheat bran and grass. A remarkable example is the comparison of the standard activity of Cellubrix $(107 \mathrm{U} / \mathrm{mL})$ and Cellulase $2000 \mathrm{~L}$ (568 U/mL) with the degradation of xylan to xylose of Cellubrix (only 50-60\%) and Cellulase 2000L (only 18-20\%). The enzyme activity towards the cellulose- and xylan-enriched fractions from grass and wheat bran was revealed to be markedly different than that found with standard assays. Therefore, the actual degradation of the xylan- and cellulose-rich fractions from wheat bran and grass could not be correlated with the (relatively low) activity of enzymes as indicated by standard assays. Therefore, in general, the choice of most suitable enzyme preparation is dependent on the substrate characteristics rather than on standard enzyme activities measured.

\section{Conclusion}

In this paper, we have presented our study on optimization of the medium and fermentation parameters for enhancing the enzyme production by B. amyloliquefaciens SS35. The statistical experimental design applied for medium optimization revealed that medium components providing carbon and nitrogen sources (viz. CMC, yeast extract, and peptone) are the most significant. The inorganic salts play relatively subordinate role. The statistical experimental design for optimization of fermentation parameters reveals the optimum range of these parameters. The optimization study of fermentation parameters reveals independent influence of the fermentation parameters, and overall, even the individual influence is only marginal, as compared to the effect of medium composition. The overall conclusion is that the enzyme production by $B$. amyloliquefaciens SS35 is mainly influenced by the medium components that supply carbon and nitrogen for the bacterial metabolism.

Acknowledgements Research funding from Department of Biotechnology, Government of India, for this work is gratefully acknowledged. The authors of this paper declare no conflict of interest of any kind.

Open Access This article is distributed under the terms of the Creative Commons Attribution License which permits any use, distribution, and reproduction in any medium, provided the original author(s) and the source are credited.

\section{References}

Apun K, Jong BC, Salleh MA (2000) Screening and isolation of a cellulolytic and amylolytic Bacillus from sago pith waste. J Gen Appl Microbiol 46:263-267

Ariffin H, Abdullah N, Umikalsom MS, Shirai Y, Hassan MA (2008) Production of bacterial endoglucanase from pretreated oil palm empty fruit bunch by Bacillus pumilus EB3. J Biosci Bioeng 106:231-236

Ballesteros M, Oliva JM, Negro MJ, Manzanares P, Ballesteros J (2004) Ethanol from lignocellulosic materials by a simultaneous saccharification and fermentation process (SSF) with Kluyveromeces marxianus CECT 10875. Process Biochem 39:1843-1848

Deka D, Bhargavi P, Sharma A, Goyal D, Jawed M, Goyal A (2011) Enhancement of cellulase activity from a new strain of Bacillus subtilis by medium optimisation and analysis with various cellulosic substrates. Enzyme Res. doi:10.4061/2011/151656 
Deka D, Das SP, Sahoo N, Das D, Jawed M, Goyal D, Goyal A (2013) Enhanced cellulase production from Bacillus subtilis by optimizing physical parameters for bioethanol production. ISRN Biotechnol. doi:10.5402/2013/965310

Gao W, Lee EJ, Lee SU, Li J, Chung CH, Lee JW (2012) Enhanced carboxymethylcellulase production by a newly isolated marine bacterium Cellulophaga lytica LBH-14 using rice bran. J Microbiol Biotechnol 22:1412-1422

Immanuel G, Dhanusha R, Prema P, Palavesam A (2006) Effect of different growth parameters on endoglucanase enzyme activity by bacteria isolated from coir retting effluents of estuarine environment. Int J Environ Sci Technol 3:25-34

Jin IH, Jing DY, Son CW, Kim SK, Gao W, Chung CH, Lee JW (2011) Enhanced production of heteropolysaccharide-7 by Beijerinkia indica HS-2001 in repeated batch culture with optimized substitution of culture medium. Biotechnol Bioprocess Eng 16:245-255

Kabel MA, van der Maarel MJEC, Klip G, Voragen AGJ, Schols HA (2006) Standard assays do not predict the efficiency of commercial cellulase preparations towards plant materials. Biotechnol Bioeng 93:56-63

Khan FABA, Husaini AASA (2006) Enhancing $\alpha$-amylase and cellulase in vivo enzyme expressions on sago pith residue using Bacillus amyloliquefaciens UMAS 1002. Biotechnol 5:391-403

Kim BK, Lee BH, Lee YJ, Jin IH, Chung CH, Lee JW (2009) Purification and characterization of carboxymethylcellulase isolated from a marine bacterium Bacillus subtilis subsp. subtilis A-53. Enzyme Microb Technol 44:411-416

Kuhad RC, Gupta R, Khasa YP, Singh A (2010) Bioethanol production from Lantana camara (red sage): pretreatment, saccharification and fermentation. Bioresour Technol 101:8348-8354

Lee YJ, Kim BK, Lee BH, Jo KI, Lee K, Chung CH, Lee YC, Lee JW (2008) Purification and characterization of cellulase produced by Bacillus amyloliquefaciens DL-3 utilizing rice hull. Bioresour Technol 99:378-386

Lee BH, Kim BK, Lee YJ, Chung CH, Lee JW (2010) Industrial scale of optimization for the production of carboxymethylcellulase from rice bran by a marine bacterium, Bacillus subtilis subsp. subtilis A-53. Enzyme Microb Technol 46:38-42

Li W, Zhang WW, Yang MM, Chen YL (2008) Cloning of the thermostable cellulase gene from newly isolated Bacillus subtilis and its expression in Escherichia coli. Mol Biotechnol 40:195-201

Lynd LR, Weimer PJ, van Zyl WH, Pretorius IS (2002) Microbial cellulose utilization: fundamentals and biotechnology. Microbiol Mol Biol Rev 66:506-577

Mutreja R, Das D, Goyal D, Goyal A (2011) Bioconversion of agricultural waste to ethanol by SSF using recombinant cellulase from Clostridium thermocellum. Enzyme Res. doi:10.4061/2011/ 340279
Nelson N (1944) A photometric adaptation of the Somogyi method for the determination of glucose. J Biol Chem 153:375-380

Pason P, Kyu KL, Ratanakhanokchai K (2006) Paenibacillus curdlanolyticus strain B-6 xylanolytic-cellulolytic enzyme system that degrades insoluble polysaccharides. Appl Environ Microbiol 72:2483-2490

Plackett RL, Burman JP (1946) The design of optimum multifactorial experiments. Biometrika 33:305-325

Rastogi G, Muppidi GL, Gurram RN et al (2009) Isolation and characterization of cellulose-degrading bacteria from the deep subsurface of the Homestake gold mine, Lead, South Dakota, USA. J Ind Microbiol Biotechnol 36:585-598

Rodhe AV, Sateesh L, Sridevi J, Venkateswarlu B, Venkateswar Rao L (2011) Enzymatic hydrolysis of sorghum straw using native cellulase produced by $T$. reesei NCIM 992 under solid state fermentation using rice straw. 3 Biotech 1:207-215

Sethi S, Datta A, Gupta BL, Gupta S (2013) Optimization of cellulase production from bacteria isolated from soil. ISRN Biotechnol. doi: $10.5402 / 2013 / 985685$

Shabeb MSA, Younis MAM, Hezayen FF, Noureldein MA (2010) Production of cellulase in low-cost medium by Bacillus subtilis KO strain. World Appl Sci J 8:35-42

Shaibani N, Ghazvini S, Andalibi MR, Yaghmaei S (2011) Ethanol production from sugarcane bagasse by means of enzymes produced by solid state fermentation method. World Acad Sci Eng Technol 59:1836-1839

Singh A, Bishnoi NR (2012) Enzymatic hydrolysis optimization of microwave alkali pretreated wheat straw and ethanol production by yeast. Bioresour Technol 108:94-101

Singh J, Batra N, Sobti RC (2001) A highly thermostable, alkaline CMCase produced by a newly isolated Bacillus sp. VG1. World J Microbiol Biotechnol 17:761-765

Singh S, Moholkar VS, Goyal A (2013) Isolation, identification and characterization of a cellulolytic Bacillus amyloliquefaciens strain SS35 from rhinoceros dung. ISRN Microbiol. doi:10.1155/ 2013/728134

Somogyi M (1945) A new reagent for the determination of sugars. J Biol Chem 160:61-68

Unrean P, Nguyen NHA (2013) Metabolic pathway analysis and kinetic studies for production of nattokinase in Bacillus subtilis. Bioprocess Biosyst Eng 36:45-56

Van Dyk JS, Sakka M, Sakka K, Pletschke BI (2009) The cellulolytic and hemi-cellulolytic system of Bacillus licheniformis SVD1 and the evidence for production of a large multi-enzyme complex. Enz Microb Technol 45:372-378

Vasudeo Z, Lew C (2011) Optimization of culture conditions for production of cellulase by a thermophilic Bacillus strain. J Chem Chem Eng 5:521-527

Zheng Y, Pan Z, Zhang R, Wang D (2009) Enzymatic saccharification of dilute acid pretreated saline crops for fermentable sugar production. Appl Energy 86:2459-2465 